\title{
Réflexions juridiques sur la notion d'expert qualifié en radioprotection : définition et responsabilités*
}

\author{
M.-C. BOEHLER**
}

(Manuscrit reçu le 28 janvier 1998, accepté le 8 avril 1998)

RÉSUMÉ La notion d'expert qualifié en radioprotection existe en droit européen depuis la première Directive EURATOM du 20 février 1959 fixant les normes de base relatives à la protection sanitaire de la population et des travailleurs contre les dangers des rayonnements ionisants. On retrouve cette notion dans toutes les versions successives de ces normes de base, et en particulier dans la dernière Directive EURATOM du 13 mai 1996. D'ici l'an 2000, date d'expiration du délai accordé par l'Union européenne pour transposer cette Directive dans les législations nationales, la France devrait normalement intégrer ce pilier du système de radioprotection dans sa réglementation révisée sous la dénomination depuis longtemps usitée de personne compétente. L'intitulé de la définition de l'expert qualifié dans la Directive de 1996 marque une évolution importante d'un point de vue juridique par l'introduction de la notion de « reconnaissance de la capacité d'agir comme expert qualifié par les autorités compétentes $»$. Cet article a pour objectif d'analyser les conséquences que pourrait avoir en France cette injonction de l'Union européenne relative à la reconnaissance de la "capacité d'agir», tant sur l'approche réglementaire des moyens à mettre à la disposition de la personne compétente, que sur l'éventuel engagement de sa responsabilité pénale.

ABSTRACT Juridical reflections on the concept of "qualified expert" in radiation protection : Definition and liability.

The concept of "qualified expert" in radiation protection exists in European law since the first version of the EURATOM Directive of the 20/02/1959 concerning the basic standards relative to the sanitary protection of the population and the workers against the dangers of ionising radiations. This concept is integrated in the successive versions of the basic standards, until the last Directive of the 13/05/1996. In the year 2000 , when the delay of transposition of this Directive into national regulations comes to an end, France should normally put this concept into its revised regulations under the name of "competent person". The definition of the qualified expert in the Directive of 1996 has been modified by the introduction of the term "capacity of acting as a qualified expert". The objective of this paper is to analyse the potential consequences in France of this "capacity of acting" on the regulatory approach of the means to be given to the qualified expert and also on his penal liability.

\footnotetext{
* Cet article a fait l'objet d'une communication orale lors du colloque organisé par la SFRP sur le thème « Personnes compétentes : retour d'expérience et perspective », Centre d'Études de Saclay, INSTN, 04/12/1997.

** Électricité de France, Secrétariat Général/Service Juridique National, 2 rue Louis Murat, 75384 Paris Cedex 08, France - Télécopie : 0140423014.
} 


\section{Introduction}

Définitions : Dans la Directive EURATOM du 15 juillet 1980 (Directive 80/836) modifiée en 1984 (Directive 84/467) et transposée dans la réglementation française en radioprotection actuellement en vigueur, l'expert qualifié est la "personne ayant les connaissances et l'entraînement nécessaires soit pour effectuer des examens physiques, techniques ou radiotoxicologiques, soit pour donner tous les conseils en vue d'assurer une protection efficace des individus et un fonctionnement correct des installations de protection, et dont la qualification est reconnue par les autorités compétentes ${ }^{(1)}$. Ce spécialiste doit donc:

- avoir préalablement suivi avec succès une formation diplômante pour dans la pratique exercer les missions assignées;

- avoir une qualification, c'est-à-dire au sens juridique « avoir une valeur suivant sa formation validée formellement par un diplôme ou toute autre attestation officielle » qui soit « reconnue par les autorités compétentes », reconnaissance qui est supposée lui conférer le statut non discutable d'un spécialiste dans le domaine de la surveillance physique.

La nouvelle Directive EURATOM du 13 mai 1996 (Directive 96/29), sur la base de laquelle la réglementation française en radioprotection est actuellement en cours de révision, ne réclame plus aux autorités compétentes la reconnaissance de la qualification mais celle de la « capacité d'agir comme expert qualifié ». L'expert qualifié est en effet désormais la "personne ayant les connaissances et l'entraînement nécessaires pour effectuer des examens physiques, techniques et radiochimiques permettant d'évaluer les doses et de donner des conseils afin d'assurer une protection efficace des individus et un fonctionnement correct des moyens de protection, et dont la capacité d'agir comme expert qualifié est reconnue par les autorités compétentes. Un expert qualifié peut se voir assigner la responsabilité technique des tâches de radioprotection des travailleurs et des personnes du public ${ }^{(2)}{ }$.

Sur la forme, la reconnaissance de la capacité d'agir réclame moins de formalisme que la reconnaissance de la qualification au sens de « formation diplômante ». Sur le fond, la notion de capacité d'agir est plus large que la notion de qualification. La formation est ici nécessaire mais insuffisante : être capable d'agir, c'est avoir les moyens suffisants pour pouvoir s'imposer afin d'atteindre les objectifs de sécurité assignés, que ces moyens soient d'ordre intellectuel, matériel ou organisationnel.

(1) Les Directives EURATOM relatives aux normes de base en radioprotection ont créé avec « l'expert qualifié » une catégorie particulière de spécialistes sui generis pour lesquels on ne trouve pas de pendant dans d'autres activités.

(2) Il y a désormais une possibilité et non l'obligation d'octroyer la responsabilité technique des tâches de radioprotection à l'expert qualifié. Celle-ci peut être attribuée à des services agrés de médecine du travail qui sont définis dans la Directive de 1996 comme suit : « organisme(s) qui peut (vent) être chargé(s) de la protection radiologique des travailleurs exposés et/ou de la surveillance médicale des travailleurs de la catégorie A et dont la qualification pour cette tâche est reconnue par les autorités compétentes ». Cette disposition ne sera pas transposée dans le système réglementaire français car elle ne correspond pas aux attributions de la médecine du travail. 


\section{La problématique de la « reconnaissance de la capacité d'agir »}

\subsection{Sur la forme}

Le choix de cette terminologie - « reconnaissance de la capacité d'agir comme expert qualifié »-dans le texte de la Directive n'est pas anodin : il correspond à une tentative de surmonter les difficultés rencontrées depuis des années en matière d'harmonisation d'une qualification formalisée de ces experts dans l'Union Européenne. Cette logique communautaire s'inspire en particulier des revendications du Royaume-Uni au moment de la préparation du texte de la Directive qui souhaitait que l'habilitation à exercer la fonction d'expert qualifié se fasse de la façon la plus pragmatique et efficiente qui soit et que soit évitée toute dérive vers la tendance trop formaliste que laissait sous entendre l'utilisation du terme de « reconnaissance de la qualification ». Le souhait est ici que l'habilitation permette de recouvrir des réalités très diverses (personne compétente en centrale nucléaire, dans la recherche, dans le domaine médical) et à l'autorité compétente de reconnaître des compétences à des degrés divers.

Le choix de ce "plus petit dénominateur commun ", à savoir la " capacité d'agir », laisse donc une souplesse assez importante d'adaptation des systèmes réglementaires nationaux à la Directive, en n'obligeant pas les états membres à prévoir la reconnaissance de titres formels et substantiels (type cursus universitaire de haut niveau par exemple). La reconnaissance est ici technico-professionnelle et donc plus générale : elle signifie que les législateurs européens ont évité de définir un niveau de formation requis qui devrait être identique dans toute l'Union européenne.

Cette analyse juridique communautaire laisse bien sûr ouvert le débat en France quant à savoir si la façon dont sont pensées les formations initiales (actuellement de quelques jours) et continue prévues par l'arrêté du 25 novembre 1987 peut correspondre à une approche qualitative suffisante de ce que l'on peut attendre en France de la part de personnes dites compétentes.

\subsection{Sur le fond}

La formation est une condition sine qua non de la qualité d'expert qualifié, mais le terme de "reconnaissance de la capacité d'agir » a une signification plus large. L'utilisation de ce terme a pour conséquence d'obliger les états membres à réfléchir à la façon de donner réglementairement à ces experts qualifiés une réelle et efficace capacité d'action. L'action de la personne compétente (sur le plan technique et administratif) correspond à la liste des missions et obligations définies dans le 


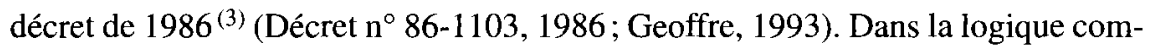
munautaire, c'est parce que l'expert a la qualification - aujourd'hui la « capacité d'agir » comme expert « et donc que les moyens lui ont été donnés pour agir - que la réglementation lui octroie ses missions d'examen technique pour l'évaluation des doses et de conseiller technique. Or, notre logique française a trop tendance à fonctionner à l'inverse, c'est-à-dire à d'abord attribuer réglementairement des missions et obligations à la personne compétente et à s'interroger seulement ensuite sur le fait de savoir si on lui a donné les moyens nécessaires pour les assumer.

La logique communautaire nous oblige ici à d'abord réfléchir sur la capacité d'agir de la personne compétente, c'est-à-dire sur les moyens matériels, intellectuels et organisationnels nécessaires et suffisants, outre la formation, pour lui permettre de remplir ses missions. Il est donc nécessaire que la réglementation française actuelle trop restreinte dans son approche de la capacité d'agir de la personne compétente soit révisée : il est actuellement seulement exigé que « la personne compétente ait préalablement suivi avec succès une formation à la radioprotection agréée par les ministres chargés du travail, de la santé et de l'agriculture », sans que soient évoqués les autres moyens nécessaires à l'accomplissement de sa tâche.

La question est ici la suivante : comment construire sa crédibilité en tant que conseiller et coordonnateur et lui accorder la capacité sur le plan humain d'agir?

\footnotetext{
(3) Placée sous la responsabilité de l'employeur et en liaison avec le C.H.S.C.T. ou à défaut les délégués du personnel, son rôle est (article 17 du décret du 2 octobre 1986) :

- d'effectuer l'analyse du poste de travail exposé sous l'angle des matériels, des procédés et de l'organisation, de telle sorte que les expositions professionnelles individuelles et collectives soient maintenues aussi bas qu'il est raisonnablement possible en dessous des limites réglementaires, et ce avec une périodicité qui est fonction du niveau d'exposition; - de veiller au respect des mesures de protection contre les rayonnements ionisants;

- de recenser les situations ou les modes de travail susceptibles de conduire à des expositions exceptionnelles ou accidentelles des travailleurs;

- d'élaborer un plan d'intervention en cas d'accident:

- d'être apte à le mettre en œuvre;

- de prendre les premières mesures d'urgence;

- de participer à la formation à la sécurité des travailleurs exposés (catégories $\mathrm{A}+\mathrm{B}$ );

- de rédiger la fiche relative aux conditions de travail (Art. 39);

- d'effectuer un contrôle après tout cas de dépassement des limites (Art. 29 Ic et II);

- d'effectuer le contrôle des sources scellées, après toute modification apportée aux modalités d'utilisation à l'équipement, aux dispositifs de sécurité ou au blindage;

- d'effectuer éventuellement le contrôle périodique fixé par l'arrêté du 20 avril 1968 (Art. 29 II);

- d'effectuer les contrôles relatifs aux sources non scellées (Ait. 30);

- de réaliser les contrôles périodiques d'ambiance (Art. 31);

- d'étudier en cas de dépassement des limites, les mesures à prendre pour remédier à toute défectuosité et en prévenir un éventuel renouvellement (Art. 35);

- de réaliser éventuellement un contrôle de contamination externe sur les travailleurs affectés dans les locaux où il fait usage de sources non scellées, au moment où ceux-ci quittent les lieux (Art. 59);

- faire procéder journellement à un contrôle de non contamination des vestiaires affectés aux travaitleurs exposés (Art. 60);

- de diriger, lors des travaux temporaires effectués à l'extérieur de l'entreprise ou de l'établissement, une ou plusieurs personnes désignées par l'employeur, chargées de veiller au respect des mesures de protection contre les rayonnements ionisants (Art. 17);

- de rédiger sous la responsabilité de l'employeur, une consigne écrite précisant l'étendue de la mission incombant à ces personnes suppléantes (Art. 17).
} 
La capacité d'agir entraîne juridiquement une obligation de moyens, moyens qui sont de façon non exhaustive (Descours, 1996) :

- outre la formation, la compétence technique et la capacité de discernement suffisante;

- l'autorité suffisante qui se traduit par un niveau hiérarchique adéquat;

- le rattachement ou l'accès direct au chef d'établissement (Lefaure et al., 1991);

- les moyens matériels (budget, de commander du matériel, pouvoir d'embaucher éventuellement...);

- et avant tout un temps significatif lui permettant d'assumer ses missions;

- mais aussi l'indépendance nécessaire à l'accomplissement de sa tâche.

C'est en fait cette notion d'indépendance qui a initialement justifié la création dans les normes de base de 1959 d'une telle personne que l'on a voulu « autonome » par rapport à l'employeur et dont la légitimité est de défendre dans l'entreprise le point de vue de la protection radiologique. Il faut évoquer ici la nécessaire indépendance de fonction de la radioprotection par rapport à l'exploitation/ maintenance. Elle doit pouvoir confronter ses contraintes aux impératifs de planning et d'exploitation, voire les imposer lorsqu'elle estime que les conditions de travail (débits de dose, risques de contamination, organisation du travail) sont inacceptables.

Quelles ont été les techniques qui juridiquement ont été utilisées pour conférer à la personne compétente un statut d'autonomie et d'indépendance, notamment par rapport à son employeur qui le nomme? D'une part, en faisant en sorte que sa qualification, aujourd'hui sa capacité d'agir, soit reconnue par les autorités compétentes (i.e. le ministère de l'Emploi et de la solidarité en France), ce qui donne à la personne compétente le statut de spécialiste non contestable par l'employeur, et d'autre part et surtout, en faisant en sorte que la réglementation lui octroie directement des responsabilités opérationnelles en matière de surveillance physique.

Dans la doctrine juridique européenne, l'expert qualifié est ainsi ce que l'on appelle en droit la longa manus (le «bras armé avancé ») de l'autorité compétente de contrôle dans l'entreprise qui agit dans l'intérêt de la radioprotection et non pas forcément dans celui de son employeur qui pourrait avoir des priorités différentes. Il est censé être ainsi une sorte de « super conseiller » qui sert de lien privilégié entre l'employeur et l'autorité de contrôle. En Allemagne, Suisse, Espagne, cela se traduit par exemple par un dialogue constant entre les responsables de la radioprotection et les autorités de contrôle, notamment lorsqu'il s'agit d'effectuer des prévisions dosimétriques (Lefaure et al., 1991). Enrico Jacchia, ancien directeur d'EURATOM et juriste, a écrit dès 1964 à propos de cette notion de longa manus : « dans les réglementations nationales relatives aux fonctions de l'expert qualifié, il serait malgré tout opportun de préciser que les fonctions de l'expert qualifié sont exercées pour le compte de l'employeur et que par conséquent celui-ci tient uniquement de l'employeur son investiture et ses pouvoirs. Ceci afin d'éviter une 
interprétation qui offrirait à l'expert le loisir de s'arroger, dans l'accomplissement de sa tâche, des pouvoirs qui sont propres aux autorités compétentes dans le domaine de la surveillance des activités nucléaires ».

\section{Responsabilité pénale}

\subsection{La responsabilité pénale sur la base du Code du Travail}

La réglementation française a repris l'idée mais pas le terme d'E. Jacchia lorsqu'elle précise que la personne compétente agit « sous la responsabilité de l'employeur », et non pas comme le conseillait E. Jacchia «pour le compte de l'employeur ». Elle n'est donc pas personnellement responsable au regard du code du travail et n'est donc en principe responsable que devant son employeur. L'employeur, responsable de l'application du code du travail dans l'établissement, en particulier de l'hygiène et de la sécurité, dont la radioprotection est un des aspects réglementés, assume la responsabilité civile et pénale de l'application de la réglementation en radioprotection. Est en effet responsable, selon le principe de base, celui qui détient l'autorité et le pouvoir pour faire appliquer les règles de sécurité (Boehler, 1998).

Or l'un des effets potentiels de la reconnaissance de la capacité d'agir est de créer une obligation de moyens qui consiste à donner à la personne compétente les moyens et l'autorité suffisants pour agir. Même si le principe fort reste celui de la responsabilité directe de l'employeur, ceci pourrait avoir pour conséquence de générer une délégation de pouvoirs sur la personne compétente. En droit, la délégation n'est admise que lorsqu'elle n'est pas totale et concerne un objet précis, et lorsqu'elle a été attribuée à un préposé investi de la compétence technique, de l'autorité et des moyens nécessaires pour veiller efficacement à l'observation des dispositions en vigueur ${ }^{(4)}$, ce qui peut tout à fait correspondre à la situation de la personne compétente.

À noter ainsi qu'en Finlande, Suède et Grande-Bretagne, le responsable radioprotection reçoit délégation de pouvoirs pour faire respecter la législation : en Finlande, la législation prévoit qu'il peut être directement cité en justice si la réglementation n'est pas respectée et dans les deux autres pays, le directeur du centre de production nucléaire est considéré comme juridiquement responsable mais le responsable radioprotection est celui qui a « la responsabilité ultime pour assurer que... » (Lefaure et al., 1991).

(4) La déleguation doit par ailleurs être expresse, acceptée et connue par le délégataire. Elle doit être précise (objet limité). II n'est pas nécessaire qu'elle soit écrite mais elle doit être notoire et accompagnée d'instructions précises. 
À partir du moment où la délégation de pouvoirs répond à ces conditions, elle entraîne, dans les limites qui lui ont été fixées, transfert de la responsabilité sur le préposé délégataire, ici la personne compétente, et donc une exonération de la responsabilité pénale de l'employeur.

Quelles seraient les peines alors encourues en droit pénal du travail en cas de non respect de la réglementation en radioprotection? Sur la base du code du travail, l'employeur ou son préposé délégataire, donc ici la personne compétente, qui enfreint par sa faute personnelle les dispositions relatives à ses obligations en matière de radioprotection encourt le risque d'être puni par le code du travail d'une amende de 25000 francs $^{(5)}$.

Le débat sur le plan juridique est clairement ici et l'enjeu devient très polémique. Si le magistrat devait interpréter ainsi le terme de capacité d'agir en considérant que les moyens et l'autorité nécessaires à l'accomplissement de sa tâche lui ont été donnés et qu'ils impliquent un transfert de la responsabilité sur la personne compétente, le risque d'interprétation pourrait être celui d'un effacement relatif de la responsabilité de l'employeur, ce qui ne serait pas souhaitable pour une bonne protection des travailleurs. Il est important que l'employeur continue de se sentir personnellement en charge des questions de radioprotection et que ce soit sous son autorité qu'agisse la personne compétente. Le principe doit être celui de l'engagement de la direction pour une diffusion large de la culture de radioprotection, qui ne doit pas être le domaine réservé de la personne compétente. Il est donc fondamental de réfléchir aujourd'hui au risque intrinsèque que comporte l'interprétation du terme de capacité d'agir et l'octroi réglementaire des moyens nécessaires à accorder à la personne compétente pour l'accomplissement de ses missions.

La future réglementation en radioprotection devrait si possible rappeler, non plus comme c'est aujourd'hui le cas dans les décrets actuels qui précisent que la personne compétente agit « sous la responsabilité de l'employeur », ce qui juridiquement peut permettre une délégation de pouvoirs, mais qu'il agit « pour le compte de l'employeur », comme le proposait très judicieusement E. Jacchia dès 1964, ce qui le dégagerait plus difficilement de sa responsabilité.

Il est capital de veiller à ce que cette notion de capacité d'agir ne dérive pas sur le plan de la responsabilité juridique et que le principe reste celui de la responsabilité forte de la direction de l'établissement. Les dérives d'une trop forte reconnaissance dans la pratique de la qualification de la personne compétente ont déjà

(5) L'amende est appliquée autant de fois qu'il y a de salariés concemés par la ou les infractions relevées dans le procès verbal dressé par l'inspecteur du travail. En cas de récidive, le juge peut prononcer un emprisonnement d'un an et/ou une amende de 60000 francs. Avant de dresser procès-verbal, l'inspecteur du travail peut inviter l'employeur à prendre toute mesure utile pour faire cesser l'infraction soit en consignant une observation préalable sur le registre prévu à cet effet, soit en confurmant la mise en demeure par lettre qui fixe un délai à l'expiration duquel ces infractions devront avoir disparu. 
par le passé été soulignées pour, si possible, mieux éclairer les choix et orientations futures. Le retour d'expérience a en effet montré que, dans un certain nombre de cas, l'existence même de personnes compétentes conduit parfois à la déresponsabilisation des employeurs qui s'en remettent à elles pour des actions qui relèvent de leur responsabilité.

\subsection{La responsabilité pénale sur la base du Code pénal}

À côté des infractions trouvant leur fondement dans le code du travail, d'autres infractions de droit commun, sur la base du nouveau code pénal entré en vigueur le $1^{\mathrm{er}}$ mars 1994, peuvent s'appliquer dans l'entreprise aux personnes physiques, voire à l'entreprise en tant que personne morale. Pour ces délits prévus par le Code pénal, ce sont toutes les personnes qui ont favorisé l'atteinte à autrui par leur négligence, imprudence, inattention, maladresse ou par l'inobservation des règlements (tous ceux qui se trouvent sur la chaîne de causalité de l'atteinte) qui sont responsables quel que soit leur niveau hiérarchique, y compris donc la personne compétente.

La personne compétente peut ainsi être jugée responsable des délits suivants qui sont en situation normale, en l'absence de tout accident du travail, l'exposition d'autrui à un risque de mort ou de blessure - la mise en danger d'autrui - (article $223-1)^{(6)}$ ou, en cas d'accident du travail, l'atteinte involontaire à la vie (article 221 $6)^{(7)}$ et les atteintes involontaires à l'intégrité physique de la personne (article 222$19)^{(8)}$. Le délit de mise en danger d'autrui semble être l'un des principaux risques pénaux de droit commun qu'encourt aujourd'hui la personne compétente.

La formation initiale et continue est ici le meilleur support préventif que l'on puisse donner à ces personnes compétentes pour, qu'outre le respect de la réglementation, elles adoptent dans l'exercice de leurs tâches toutes les précautions susceptibles de les prémunir contre l'éventuelle sanction de leur responsabilité. Cette formation doit aussi leur permettre de mieux comprendre leur éventuelle responsabilité pénale et civile et en conséquence dans le même temps d'éclairer au mieux leur capacité de discernement et de s'acquitter de façon optimale de leurs missions. Une réflexion devrait être amorcée sur le contenu du tronc commun de formation

(6) Le délit de mise en danger d'autrui par la violation d'une de ses obligations en radioprotection est puni d'un an d'emprisonnement et de 100000 francs d'amende.

(7) L'atteinte involontaire à la vie est punie d'une peine de 3 ans d'emprisonnement et de $300000 \mathrm{~F}$ d'amende. Le nouveau code pénal a prévu une circonstance aggravante en cas de manquernent délibéré à une obligation de sécurité qui porte la sanction à 5 ans d'emprisonnement et $500000 \mathrm{~F}$ d'amende.

(8) Pour les atteintes involontaires à l'intégrité physique, le nouveau code pénal prévoit des peines de 2 ans d'emprisonnement et de $200000 \mathrm{~F}$ d'amende, et en cas de manquement délibéré des peines plus lourdes de 3 ans d'emprisonnement et $300000 \mathrm{~F}$ d'amende. Lorsque l'incapacité totale de travail est inférieure ou égale à trois mois, l'amende est de $10000 \mathrm{~F}$. Si elle résulte d'un manquement délibéré, le responsable est passible d'un an de prison et de $100000 \mathrm{~F}$ d'amende. En cas de récidive, toutes les peines précitées sont doublées. 
initiale prévu par l'arrêté du 25 novembre 1987 qui porte sur les aspects réglementaires et organisationnels de la radioprotection et envisager d'insister davantage sur les aspects de responsabilité pénale qui peuvent toucher directement la personne compétente.

\section{Conclusion}

En résumé, il est nécessaire de donner réglementairement à la personne compétente les moyens intellectuels, matériels et organisationnels d'agir (formation, autorité...) pour répondre à l'injonction communautaire relative à la capacité d'agir de l'expert qualifié. Dans le même temps, l'attribution de ces moyens peut créer, par une délégation de pouvoirs, un transfert de responsabilité de l'employeur sur la personne compétente. L'employeur ne doit cependant pas être désengagé de ses responsabilités en matière de radioprotection. Dans la future réglementation française, il faudrait donc à la fois donner les moyens d'agir à la personne compétente, et dans le même temps, préciser que la personne compétente agit pour le compte de l'employeur afin d'éviter le plus possible toute dilution de la responsabilité à terme préjudiciable à une bonne protection des individus.

Au vu des nombreuses questions qu'amènent l'interprétation juridique de la capacité d'agir, sa traduction sur le plan pratique et ses conséquences en matière de responsabilité (et des nombreux débats qui ne manqueraient pas de se développer en conséquence devant un tribunal), il est donc aujourd'hui plus fondamental encore d'être vigilant dans la formulation de la future réglementation en radioprotection de notre pays sur cette question.

L'injonction européenne d'octroyer à la personne compétente une réelle capacité d'agir correspond à une montée en puissance de la personne compétente. Ce rôle accru que l'on donne à ces personnes garantes sur le terrain de la relation sourcesexpositions des travailleurs est une réponse possible au renforcement du principe ALARA dans le système de protection radiologique, dont elles sont les leviers tant dans la réglementation que dans la pratique. Parce qu'elles ont ce rôle fondamental, la future réglementation doit tendre à mettre en place les garanties juridiques suffisantes pour que ces personnes puissent disposer des moyens d'agir et de prendre les initiatives nécessaires à la démarche de qualité que suppose le principe ALARA, tout en étant dégagées le plus possible de la pression psychologique que peut représenter la mise en jeu de leur responsabilité sur le plan individuel.

\section{Références}

Boehler M.C. (1998) Problèmes de responsabilité pénale dans le domaine de la protection radiologique, Cours du DESS de Radioprotection-Radiopathologie, INSTN, 14 p.

Descours C. (1996) Rapport au Premier Ministre sur la Sécurité des personnels utilisant des appareils à rayonnements ionisants dans les établissements de santé, Rapport de $\mathrm{M}$. Charles Descours, 
Sénateur de l'Isère, Parlementaire en mission, $146 \mathrm{p}$.

Décret $n^{\circ} 86-1103$ du 2 octobre 1986 relatif à la protection des travailleurs contre les rayonnements ionisants hors des installations nucléaires de base, JORF du 12/10/1986, modifié par le décret $\mathrm{n}^{\circ}$ 88-662 du 6 mai 1988, JORF du 08/05/1988.

Directive 80/836/EURATOM du Conseil du 15 juillet 1980 portant modification des Directives fixant les Normes de base relatives à la protection sanitaire de la population et des travailleurs contre les dangers résultant des rayonnements ionisants, JOCE L-246-1 du 17/09/1980, p. 1.

Directive 84/467/EURATOM du Conseil du 3 septembre 1984 modifiant la Directive 80/836/EURATOM en ce qui concerne les normes relatives à la protection sanitaire de la population et des travailleurs contre les dangers résultant des rayonnements ionisants, JOCE L-265-4 du 05/10/1984, p. 4.

Directive 96/29/EURATOM du Conseil du 13 mai 1996 fixant les Normes de base relatives à la protection sanitaire de la population et des travailleurs contre les dangers résultant des rayonnements ionisants, JOCE L-159 du 29/06/1996, p. 1.

Geoffre B. (1993) La radioprotection à l'intention des personnes compétentes - Cours relatif à la législation et à la réglementation : rôle de la personne compétente, INSTN, $37 \mathrm{p}$.

Lefaure C., Benedittini M. (1991) Caractéristiques de l'organisation de la radioprotection dans des centres de production nucléaires à l'étranger, Rapport CEPN $\mathrm{n}^{\circ} 186,17 \mathrm{p}$. et annexes. 